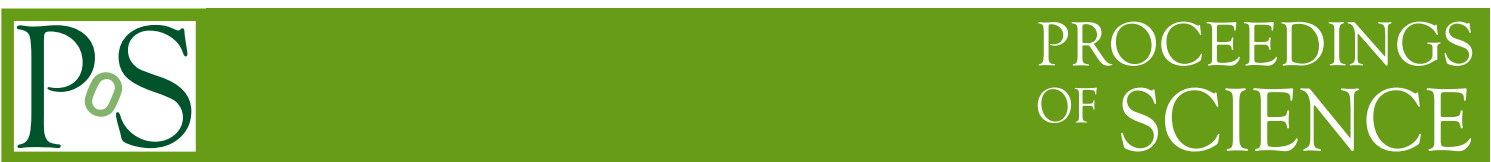

\title{
Cosmic Ray propagation uncertainties and Dark Matter
}

\author{
Richard Taillet ${ }^{* \dagger}$ \\ LAPTH, Université de Savoie, CNRS \\ BP110, F-74941 Annecy-le-Vieux Cedex, France \\ E-mail: tailletelapp.in2p3.fr
}

\begin{abstract}
Dark matter particles could be detected indirectly through their annihilation into particles of the standard model, like antiprotons or positrons. This is a very challenging program for several reasons. First, one has to understand all the astrophysical processes that could also lead to the production of these antiprotons or positrons. Second, to characterize an excess it is necessary to understand the background signal, due to spallations of cosmic rays on the interstellar medium. This requires to fully understand the propagation of these charged particles in the Galaxy. Propagation is described by a diffusion equation, with other physical effects such as spallation, energy losses, diffusive reacceleration and convection. The parameters entering this equation are degenerate and there is no "standard model for cosmic ray diffusion". As a result, the studies of indirect detection through antimatter must take into account the studies of propagation of cosmic-ray nuclei (which give constraints on the propagation parameters), which is not actually the way is is always done. Moreover, comparison of the exotic signal to the standard background must be done within the same framework, using the same equation diffusion, which unfortunately is also not actually the way is is always done.
\end{abstract}

Identification of Dark Matter 2010-IDM2010

July 26-30, 2010

Montpellier France

* Speaker.

${ }^{\dagger}$ A footnote may follow. 


\section{Introduction}

Many candidates for particle dark matter could be detected in an indirect way, through their annihilation or decay signal into standard particles, like protons,electrons, electrons, neutrinos, antiprotons or positrons. The observation of an excess of any of these particles could be due to dark matter annihilation and demands a thorough analysis of all the standard explanations for the excess. Such an excess should be more readily observable for antiprotons and positrons, that are pretty rare species (antiprotons are $10^{4}$ times less abundant than protons in cosmic rays). This is why large efforts are made to study the antiproton and positron fluxes in cosmic rays.

In the context of indirect detection of dark matter, these studies are twofolds. First, the contribution due to standard astrophysical processes (acceleration in sources and spallation) must be computed. This is performed in a chosen theoretical framework, taking into account the physical effects that are believed to be relevant, for instance diffusion models with large-scale convective wind, diffusive reacceleration, energy losses and spallative destruction of some species leading to the creation of others. Second, the contribution due to the annihilation or the decay of dark matter particles must alos be computed, once an hypothesis has been made about the nature of dark matter, leading to a specific underlying particle physics model.

The sources of antiprotons or antiprotons are different in both cases, but their propagation of cosmic rays obeys the same physics, in the standard case and in the exotic case. However, in many cases, published studies focus on one of these two aspects only. This is a problem when one tries to compare a predicted exotic flux, computed with a given diffusion model, and then compares it to a standard flux, computed with another diffusion model. Ideally, the two studies should be made altogether, or at least using the same propagation code. Ignoring this fact has been the cause for some confusion. Even comparing a predicted exotic flux to the observed flux to exclude the dark matter model when the first is larger than the second is tricky: the exotic can depend strongly on the propagation parameters, which are not know independently from each other (see below).

Charged particles propagating in the Galaxy interact with the interstellar magnetic field. This magnetic field can be splitted into a regular component, with spatial variations on scales of the order of galactic visible features (arms, bulge, disc), and a turbulent component, showing spatial variations on a large range of scales, from a fraction of kiloparsec to a fraction of parsec. The two component have similar amplitudes, and the latter is responsible for a propagation of charged particles that is of diffusive nature. The diffusion coefficient depends on the energy. At a given energy, it is directly related to the power spectrum of the spatial distribution of the magnetic field at the scale corresponding to the Larmor radius at that energy. Unfortunately, our knowledge of the Galactic magnetic field is not acurate anough to be able to deduce the diffusion coefficient from observations. Theoretical approaches give indications about the kind of turbulence that might be present in our Galaxy, and in several cases the diffusion coefficient is given by

$$
K(E)=K_{0}\left(\frac{\mathscr{R}}{\mathscr{R}_{0}}\right)^{\delta}
$$

where $\mathscr{R}$ stands for the magnetic rigidity (defined as the ratio between linear momentum and charge, $p / q), K_{0}$ the normalization, $\delta$ a spectral index depending on the kind of magnetic tur- 
bulence ( $\delta=0.3$ is known as Kolmogorov turbulence, but other turbulences exist, with values of $\delta$ typically in the range $0.3-1$ ).

The study of cosmic ray nuclei (no exotic contribution is expected) give important information about these two parameters $K_{0}$ and $\delta$ as we will see below, but it does not give precise values for them: most studies only provide relations between this parameters, regions of the parameter space that are consistent with observed cosmic rays abundances and spectra. The spatial distribution of exotic sources is very different from that of standard sources. As a result, the sets of parameters giving similar standard fluxes can lead to very different exotic fluxes. To summarize : determining the diffusion parameters from the study of standard nuclei does not provide a unique prediction for exotic species.

\section{Physics of cosmic ray propagation}

During their propagation in the Galaxy, charged particles interact with the interstellar medium. The inhomogeneities of the magnetic field are responsable for spatial diffusion, which also implies confinment. They also lead to diffusion in momentum space, which is called diffusive reacceleration. Particles also interact with the matter present in the interstellar medium. This can lead to energy losses and to spallation, i.e. nuclear reactions leading to the breaking of nuclei into smaller nuclei. Finally, the diffusive medium is not at rest with respect to the Galaxy, il is blown away because of the stellar activity of the disk. Cosmic rays are carried away from the disk by convection. Each of these physical effects is described by one or several parameters :

- diffusion : $K_{0}$ and $\delta$;

- diffusive reacceleration : Alfvén velocity $V_{a}$;

- convection : velocity $\vec{V}(\vec{r})$. Assuming uniformity for the wind, this boils down to a single parameter $V_{c}$;

- geometry of the galaxy : radius and height of the galactic disk, height $L$ of the diffusive halo if it is modelled as a cylinder;

- spallation : spatial distribution of interstellar matter : $n_{\mathrm{ISM}}(\vec{r})$. For most purposes, a coarsegrained version where cylindrical symmetry is assumed is sufficient, $n_{\mathrm{ISM}}(r)$. It is even often a good approximation to consider that $n_{\mathrm{ISM}}$ is uniform in the Galactic disk;

- spallation : nuclear cross-sections.

Some of these parameters, like the density of the interstellar medium or the spallation cross section, can be supposed to be known, to some extent (even though there may be sizeable uncertainties or discrepancies between differents measurements). Others, like the normalisation $K_{0}$ of the diffusion coefficient or the height $L$ of the diffusion halo, suffer from large uncertainties.

The value of these parameters can be constrained by studies of cosmic-ray nuclei abundances and elemental ratios. In particular, the energy dependance of the boron-to-carbon $(\mathrm{B} / \mathrm{C})$ ratio is sensitive to these parameters. Several studies, in particular those done by the USINE team, have determined the regions of the parameter space that are consistent with the observed $\mathrm{B} / \mathrm{C}$ ratio 

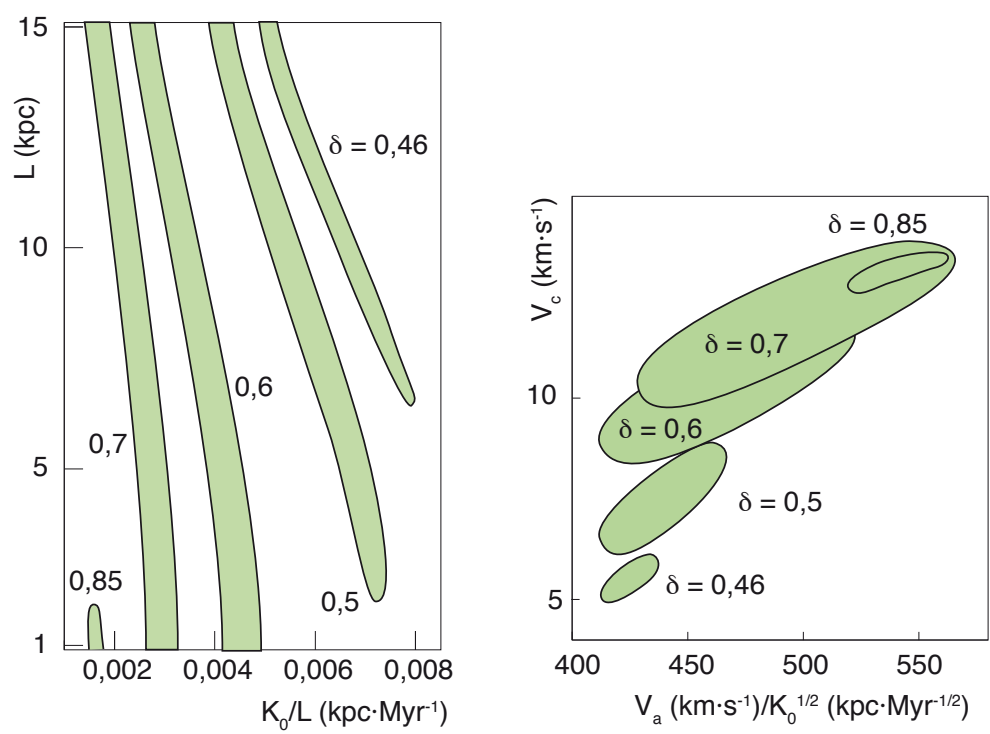

Figure 1: Regions of the parameter space that are "allowed", i.e. consistent with the observed B/C ratio (from [1]).

$[1,2,3]$. The corresponding parameters will be called "allowed parameter" in the following (see Fig. 1).

We insist on the fact that the study of nuclei does not provide only one set of propagation parameters, but a whole continuum of them, with very different values. For instance, the height of the diffusive halo is very poorly constrained, and could be as small as $1 \mathrm{kpc}$ or larger than $20 \mathrm{kpc}$.

\section{Indirect detection of dark matter through antimatter}

If dark matter is made of exotic particles, they can annihilate into particles of the standard model, and could be detected as an excess in the observed fluxes and spectra of these particles. It is more promising to look for an exotic contribution in the antiproton and positron fluxes, are the standard background over which it must be compared is lower.

Standard antiprotons are purely secondary, i.e. created by spallation of protons or helium nuclei on interstellar matter. The standard production term for antiprotons is thus pretty well know, once the density of interstellar matter, the flux of cosmic-ray protons and the spallation crosssections are known, provided the high-energy physics describing the formation of antiproton nuclei is well understood. The fact that the propagation parameters can take many allowed values translates into an uncertainty band in the computed spectrum of standard antiprotons (see Fig. 2). This band is rather narrow, this is because antiprotons are secondary species, they are sensitive to the propagation parameters in a very similar way as the secondary nuclei used to constrain them.

When the same propagation parameters are applied to the antiprotons of exotic origin, the uncertainty on the computed flux is much larger, as these exotic species are sensitive to these parameters in a different way than secondary nuclei. It is thus impossible, given a particle physics model with specified annihilation cross-sections and branching ratios, to determine a unique propagated spectrum and to make a definite prediction. What is possible is to provide a band containing 

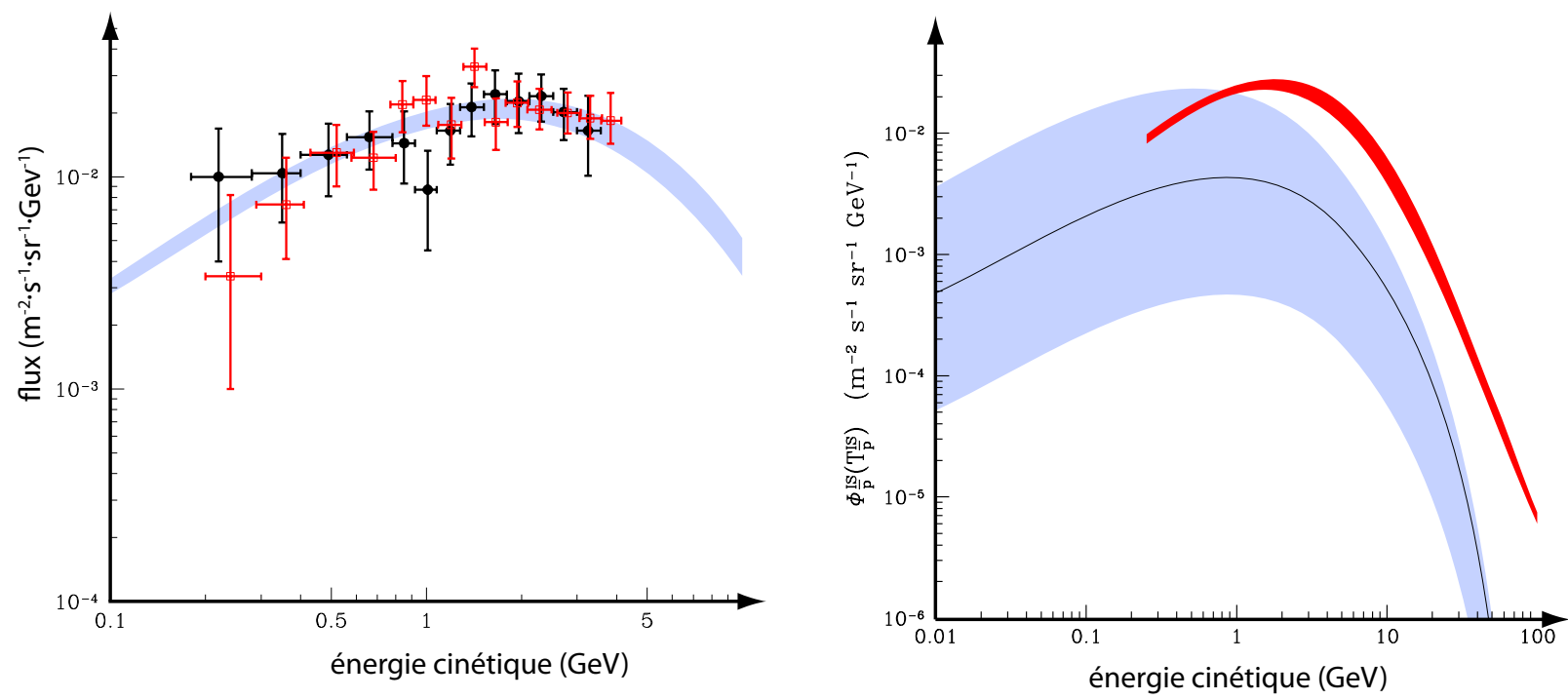

Figure 2: Uncertainty band on the antiproton spectrum due to the degeneracy of the propagation parameters: for the standard component on the left (from [4]) and for an example of exotic component on the right (from [5]).

all the spectra obtained with the parameter sets consistent with B/C. Ideally, one should compute the exotic source term, propagate the exotic antiprotons and the standard antiprotons with the same diffusion model, and see if some of the allowed parameter set give a spectrum that fit the observations. As the standard component alone can explain the data, this procedure only exclude some dark matter candidates.

The propagation of positrons is very different from that of antiprotons : it is dominated by energy losses. However, the same general remarks hold: a/ the degeneracy of the propagation parameters gives a band instead of a single curve for the theoretical prediction (see Fig.3) and b/ one should study the exotic component and the standard component with the same diffusion model.

Before claiming that some data exhibits an excess, it is very important to state over which background trhe observed feature is an excess. Recently, the PAMELA collaboration found that the positronic fraction $\left(e^{+} /\left(e^{+}+e^{-}\right)\right)$was rising sharply with energy, above a few $\mathrm{GeV}$, and this has been called an excess, because this rise did not follow one theoretical curve labelled "the prediction of the standard cosmic rays propagation model". However, there is no such thing as a standard model, as we stressed before. Instead, there are many sets of allowed parameters. To unravel an excess, one should check that the data are consistent with no propagation model, whatever the values for the parameters (inside the allowed values), and not that it is merely inconsistent with one of them.

\section{Conclusion}

Indirect detection of dark matter through charged particles (antiprotons and positrons) could be a good way to actually dark matter in a way that is not gravitational. However, one must pay 

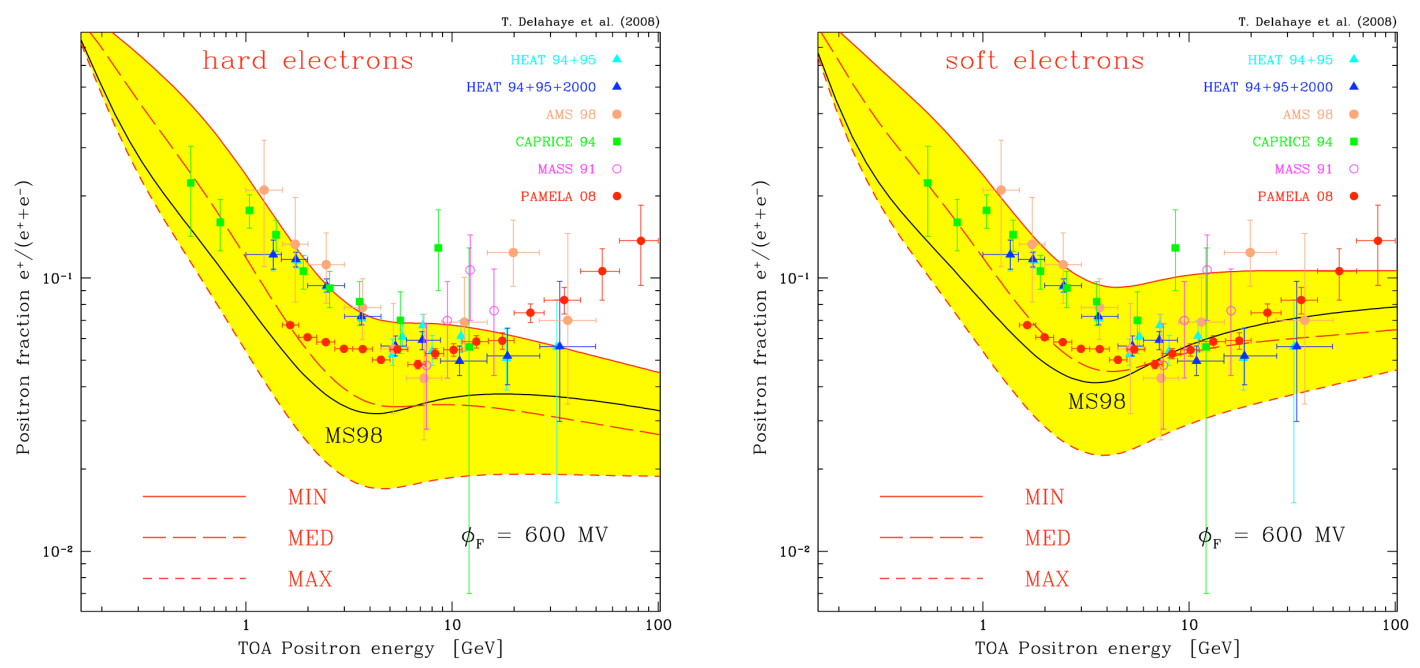

Figure 3: Uncertainty band on the positron spectrum due to the degeneracy of the propagation parameters: for a hard spectrum (left) and for a soft spectrum (right) (from [6]).

attention to the two following (depressing) remarks :

- a negative result (the observed flux is consistent with some theoretical expectation from standard physics) is very difficult to translate into an exclusion of a dark matter candidate : because of the uncertainties in the diffusion parameters (due to the degeneracy of these parameters), one can only exclude combinations of particle physics parameters and propagation parameters, in many cases;

- a positive result (the observed flux is higher than any theoretical expectation) could simply mean that there is a primary source that has not been taken into account. This could be the case for the PAMELA result as shown in [7].

This latest point is very crucial : positive indirect detection of dark matter through antimatter would require an exhaustive understanding af all astrophysical processes that could also lead to an excess, as well as a excellent knowledge of the propagation parameters. This is probably one of the most challenging way to detect dark matter.

\section{References}

[1] Maurin, D. ; Donato, F. ; Taillet, R. ; Salati, P. ; The Astrophysical Journal 555 (2001) 585-596

[2] Maurin, D. ; Taillet, R. ; Donato, F. Astronomy and Astrophysics 394 (2002) 1039-1056

[3] A. Putze, L. Derome, D. Maurin ; Astronomy \& Astrophysics 516 (2010) A66

[4] Donato, F. ; Maurin, D. ; Salati, P. ; Barrau, A. ; Boudoul, G. ; Taillet, R. ; The Astrophysical Journal 563 (2001) 172-184

[5] Barrau, Aurélien ; Salati, Pierre ; Servant, Géraldine ; Donato, Fiorenza ; Grain, Julien ; Maurin, David ; Taillet, Richard ; Physical Review D72 (2005) id. 063507 
[6] Delahaye, T.; Lineros, R. ; Donato, F. ; Fornengo, N. ; Lavalle, J. ; Salati, P. ; Taillet, R. ; Astronomy and Astrophysics 501 (2009) 821-833

[7] Delahaye, T.; Lavalle, J.; Lineros, R.; Donato, F.; Fornengo, N. ; Astronomy \& Astrophysics 524 (2010) 51 